\title{
The importance of gap states for energy level alignment at hybrid interfaces
}

\author{
D.A. Racke, L.L. Kelly, O.L.A. Monti* \\ Department of Chemistry and Biochemistry, University of Arizona, Tucson, AZ \\ *e-mail: monti@u.arizona.edu \\ Phone: +15206261177
}

\begin{abstract}
Energy level alignment and electronic structure at organic semiconductor interfaces must be controlled to ensure efficient carrier harvesting or injection in next-generation organic optoelectronic technologies. In this context, hybrid organic/inorganic semiconductor interfaces exhibit particularly rich physics. Here, we show that states in the band gap of the inorganic layered van der Waals dichalcogenide $\mathrm{SnS}_{2}$ play an important role in determining energy level alignment at the hybrid interface with copper phthalocyanine (CuPc). By taking advantage of the closely related CuPc film growth on $\mathrm{SnS}_{2}$ and the well-studied interface of CuPc/HOPG, we are able to trace spectroscopic differences to the fundamentally different electronic interactions across the two interfaces. We provide a detailed picture of the role of gap states at the hybrid interface and shed light on the electronic properties of inorganic semiconductors in general and metal dichalcogenides in particular.
\end{abstract}




\section{Introduction}

The energy level alignment at organic semiconductor interfaces is a critical factor in determining the performance of organic optoelectronic devices. There are many elements that establish this alignment [1-4], and a predictive and quantitative understanding of these interfaces has remained elusive, despite a great deal of progress in elucidating and modeling the electronic structure of organic thin films. In particular, states with energies in the band gap of the organic semiconductor have recently drawn significant attention as an important factor in determining charge-injection or -extraction barriers [3-6]. Here, we extend this growing understanding to the hybrid heterointerface between organic and inorganic semiconductors. We show how gap states in the inorganic semiconductor also develop a significant influence on the interfacial energy level alignment, often in ways that are not expected from current models for organic semiconductor interfaces.

We compare the interfaces developed by the prototypical organic semiconductor copper phthalocyanine (CuPc) on the quasi-2D layered materials tin disulfide $\left(\mathrm{SnS}_{2}\right)$ and highly ordered pyrolytic graphite (HOPG). These substrates are structurally related but exhibit fundamentally different electronic properties near the Fermi level, ideally suited to highlight the influence of substrate gap states on energy level alignment. Comparison between $\mathrm{CuPc}_{\mathrm{SnS}}$ and $\mathrm{CuPc} / \mathrm{HOPG}$ is facilitated by seminal work on CuPc/HOPG and related systems by the group of Ueno [5-12], enabling us to reveal the different physics that emerge at organic/inorganic hybrid interfaces.

In the spirit of a festschrift article, and to honor these contributions, we start by briefly reviewing salient features of interfaces of phthalocyanines on layered surfaces. For a more general description of molecular behavior at interfaces, we refer to several recent publications $[4,13,14]$. Taking advantage of the atomically flat surface of layered materials such as HOPG and the transition metal dichalcogenides (TMDs), considerable effort has been spent on establishing the growth and structure of the molecular film. Dispersion interactions are typically much weaker across this type interface than e.g. 
on coinage metal surfaces $[15,16]$; as a result, the molecular film structures on different layered van der Waals materials resemble each other strongly. Indeed, the thin film structure of various phthalocyanines has been compared on molybdenum disulfide $\left(\mathrm{MoS}_{2}\right)$ and tin disulfide $\left(\mathrm{SnS}_{2}\right)$ using techniques such as angle-resolved ultraviolet photoemission spectroscopy (ARUPS) and low-energy electron diffraction (LEED) $[7,9,17-19]$. Such structural understanding is essential for a correct interpretation of the interfacial spectroscopy as revealed e.g. by photoelectron spectroscopies $[20,21]$.

Large workfunction drops observed at the interface of phthalocyanines and TMDs reflect a strongly perturbed electronic structure and may in fact obscure some of the more subtle interactions $[10,22]$. HOPG provides however a highly suitable structural analog to TMDs, promoting nearly identical film growth while providing an electronically more inert surface. As a result, the interfacial electronic structure developed at organic/HOPG interfaces reveals a wide range of phenomena previously concealed in the initial investigations on TMDs. Very narrow ultraviolet photoemission peak widths were observed for CuPc/HOPG. If interpreted as homogeneous linewidths, these measured peak widths enable the calculation of photohole lifetimes, providing a first principles estimate of carrier mobilities at the interface [8]. Growth of an ordered layer of dipolar titanyl phthalocyanine (TiOPc) was shown to create a uniform electrostatic field at the interface [11], which enables extrinsic control of the surface electronic structure $[11,23]$. This theme has since then been picked up more generally and extended to a broad class of self-assembled monolayers [24-27].

The exquisite spectroscopic resolution available for interfaces of organic semiconductors with HOPG has been instrumental in uncovering the importance of gap states in determining interfacial energy level alignment. First indications in this direction were obtained from the discovery of a reversible phase transition for CuPc at $130 \mathrm{~K}$ on HOPG, which alters the electronic structure at the interface [28]. Further details regarding the role of gap states were revealed by using layered van der Waals materials, such as $\mathrm{MoS}_{2}$, as a template for ultraflat metallic substrates, as e.g. achieved by 
growing a thin layer of indium [29]. Metastable atom electron spectroscopy (MAES) of perylene3,4,9,10-tetracarboxylic dianhydride (PTCDA) on such a structure revealed strong interfacial interactions due to gap states induced in PTCDA [29], also observed for other molecule/metal systems [30,31]. Strikingly, defects in molecular packing of pentacene on HOPG were demonstrated to control the energy level alignment, explained by a perturbation of the pentacene highest occupied molecular orbital (HOMO) and consequently a change of the conditions for Fermi level pinning [32]. The importance of gap states in the organic thin film was made explicit with their reversible creation by exposure to inert gas, suggested to penetrate and alter the thin film structure $[5,6,33]$. These experiments demonstrate that the energy level alignment may be controlled by a relatively low density of states (DOS) in the organic thin film. This density of gap states (DOGS) pins the molecular states at the Fermi energy and thus limits the extent to which carrier injection barriers can be minimized. The DOGS is typically quite small and hence still rather challenging to detect $[5,6,32,33]$, thus obscuring more detailed insight into the origins of energy level alignment at organic/metal interfaces.

Therefore, an understanding of the electronic structure of organic semiconductor interfaces that incorporates the influence of a DOGS provides a powerful basis for predicting energy level alignment. Some essential ideas in this context were recently summarized by Oehzelt et al. [3]. One of the primary conclusions from this body of work is that the charge injection/extraction barrier can be minimized by reducing the DOGS in the organic semiconductor film, which reduces the propensity for Fermi level pinning. In addition to minimizing the organic semiconductor gap states arising from e.g. structural packing/defects $[5,6,32,33]$, the DOGS can also be reduced by attenuating electronic coupling to metallic electrodes [3].

Assuming for the sake of simplicity similar interfacial electronic coupling matrix elements between substrate and organic semiconductor, replacing the metallic substrate with an inorganic semiconductor might therefore be expected to result in improved energy level alignment. This stems 
from a typically much reduced substrate DOS vis-à-vis clean metal surfaces. This prediction is however not born out experimentally, even for the quasi-2D transition metal dichalcogenides (TMDs) that present atomically flat and inert surfaces and generally substantial bandgaps in the range of 1-2 eV. Hydrogen and lead phthalocyanine $\left(\mathrm{H}_{2} \mathrm{Pc}\right.$ and $\left.\mathrm{PbPc}\right)$ each establish significant interface dipoles when deposited on $\mathrm{MoS}_{2}[10,22]$, despite the fact that the ionization energy of these molecules is in excess of the substrate workfunction. This implies the existence of strong interfacial interactions despite the presumed low DOS in the $\mathrm{MoS}_{2}$ bandgap. Furthermore, the interfacial electronic structure of $\mathrm{MoS}_{2}$ was observed to depend significantly on the surface preparation [10], demonstrating that the electronic character of the TMD surface is rather sensitive to structural effects. Based on previous work in a related system, these observed interfacial interactions may in fact arise predominately from a finite DOGS in the inorganic semiconductor substrate rather than in the organic thin film [34]. Indeed, groundstate interfacial charge transfer between CuPc and substrate defects has previously been demonstrated to control energy level alignment at the hybrid CuPc/indium phosphide (InP) interface [35]. Although the relevance of this mechanism for the inert TMD surfaces is somewhat surprising, it is also supported by the observation of defect-induced carrier scattering and trapping near the TMD surface [36,37], dynamic shifts in energy level alignment at TMD interfaces [34], and electronic coupling between different stacked van der Waals materials [38].

To explore this phenomenon and elucidate the role of these gap states on the interfacial energy level alignment, we compare here the interfacial electronic structure of CuPc on the two related layered materials HOPG and $\mathrm{SnS}_{2}$. HOPG is a layered semimetal, and the CuPc/HOPG interface has been studied extensively as a model of a nonreactive organic semiconductor interface $[8,11,28,39,40]$. We therefore use this system as a reference for the more electronically active surface of the layered semiconductor $\mathrm{SnS}_{2}$. Since film structure can play an important role in the electronic structure at organic semiconductor interfaces, we have deliberately chosen these substrates to provide as similar growth modes as possible 
while varying the electronic nature of the substrate. Previous research has already established the high degree of similarity of molecular growth on $\mathrm{SnS}_{2}$ and HOPG for several different organics, showing that both surfaces support Stranski-Krastanov growth [41,42]. This is consistent with our spectroscopic observations presented below as well. Moreover, this conclusion is further supported by STM studies of CuPc monolayers on $\mathrm{MoS}_{2}$ and HOPG, which indicate similar packing structures and unit cells for both interfaces $[43,44]$. Therefore, one may anticipate that significant differences observed between $\mathrm{CuPc} / \mathrm{HOPG}$ and $\mathrm{CuPc} / \mathrm{SnS}_{2}$ should be attributed to changes in the interfacial electronic interactions, rather than to trivial differences in the organic thin film growth. We will revisit this proposition at several points in the remainder of our report, demonstrating how differences in film structure are insufficient to explain the observed phenomena.

After a brief summary of experimental details, we compare the interfacial energy level alignment for CuPc/HOPG and CuPc/SnS 2 . We observe that HOPG provides an inert substrate with virtually no vacuum level offset and thus a rather low DOGS. In contrast, the CuPc/SnS 2 interface displays interactions that can only be fully explained by a finite DOGS in the substrate. Finally, we discuss the nature and identity of these observed gap states for $\mathrm{SnS}_{2}$.

\section{Materials and Methods}

All photoelectron spectra were collected at room temperature in a VG EscaLab MK II

photoelectron spectrometer, base pressure $2 \times 10^{-10}$ Torr, with a He(I) lamp (Specs UVS 10/35, 30 angle of incidence from normal) as an excitation source. Spectra were referenced to the Fermi energy of the analyzer, which was calibrated using a sputter-cleaned Au foil. The sample workfunction was measured from the baseline intercept with the spectral slope at the inflection point of secondary electron cutoff (SECO); a -5 V bias was applied for workfunction measurements to obtain a clear SECO. Spectra were recorded with a takeoff angle of $0^{\circ}$ with respect to surface normal, $\pm 12.5^{\circ}$ acceptance angle, $5 \mathrm{eV}$ pass energy, and no bias voltage applied. 
Bridgman-grown $\mathrm{SnS}_{2}$ samples were prepared according to previously published methods [45]. The carrier concentration of the doped crystal was determined to be $2.3(1) \times 10^{17} \mathrm{~cm}^{-3}$ by the van der Pauw method [45]. Immediately prior to introduction into an ultrahigh vacuum loadlock, base pressure of $1 \times 10^{-9}$ Torr, the $\mathrm{SnS}_{2}$ sample was cleaved in air with cellophane tape. After pumping down, the sample was transferred to the photoelectron spectrometer, equipped with an integrated sample heater. The sample was degassed and cleaned by annealing at $\sim 250^{\circ} \mathrm{C}$ for approximately 12 hours.

Highly ordered pyrolytic graphite (HOPG) samples were purchased from SPI (grade SPI-1). As with the $\mathrm{SnS}_{2}$ samples, the HOPG substrate was cleaved in air, introduced into the ultrahigh vacuum system and annealed at $\sim 450{ }^{\circ} \mathrm{C}$ for approximately 12 hours.

Copper phthalocyanine (CuPc) was purchased from Sigma-Aldrich and purified by three cycles of gradient sublimation. Prior to deposition from a home-built Knudsen cell, the cell was degassed by ramping from $100{ }^{\circ} \mathrm{C}$ to $360{ }^{\circ} \mathrm{C}$ over three hours. The cell was then maintained at $360{ }^{\circ} \mathrm{C}$ for approximately 30 minutes to establish a consistent deposition rate. Vanadyl naphthalocyanine (VONc) was purchased from Sigma Aldrich and used without further purification.

Molecular films were deposited onto room-temperature substrates at $1 \AA / \mathrm{min}$ in a custom-built deposition chamber, base pressure of $1 \times 10^{-9}$ Torr. Film thicknesses were monitored using a quartz crystal microbalance that was calibrated against known thicknesses for molecular films on HOPG. $3 \AA$ of CuPc constitutes one monolayer equivalent (1 MLE), in agreement with previous experiments [40]. The molecular films were not annealed following deposition.

\section{Results}

We begin by examining the growth mode of the CuPc molecules on both HOPG and $\mathrm{SnS}_{2}$. The vanishing DOS of HOPG near the Fermi energy $\underline{E}_{\underline{F}}$ results in clearly visible molecular features in the UP spectra already at low coverages, as shown in Figure 1. The same features can also be identified in the 
CuPc/SnS 2 spectra (Figure 1B), accompanied however by strong $\mathrm{SnS}_{2}$ valence band (VB) features at binding energies greater than $2 \mathrm{eV}$. No $\mathrm{SnS}_{2}$ gap states are detectable via UPS on bare $\mathrm{SnS}_{2}$; the small DOS observed at binding energies between 2 and $0.5 \mathrm{eV}$ on bare $\mathrm{SnS}_{2}$ is due to photoemission from the Hel( $\beta$ ) satellite. In both systems, the pronounced spectral changes upon deposition of approximately 1 MLE suggest that the initial deposition results in mono- or few-layer islands, in agreement with previous studies on related systems $[43,44]$. However, after 1 MLE coverage, further deposition results in minimal changes to the spectral appearance, with the intensity of the substrate peaks decreasing only slowly. Thus, even at $>3 \mathrm{MLE}$ coverage of $\mathrm{CuPc}$, the $\sigma^{*}$ peak for HOPG and the $\mathrm{S} 3 p$ peaks for $\mathrm{SnS}_{2}$ remain prominent. This suggests strongly, that CuPc under these growth conditions form 3D islands that grow predominately in the vertical direction. These observations are consistent with a wide variety of experiments that detail molecular growth modes on both HOPG and TMDs $[8,11,39,41,42]$. Most importantly, the growth mode seems to be very similar for both substrates, thus allowing isolation and careful analysis of the differences in electronic structure. 

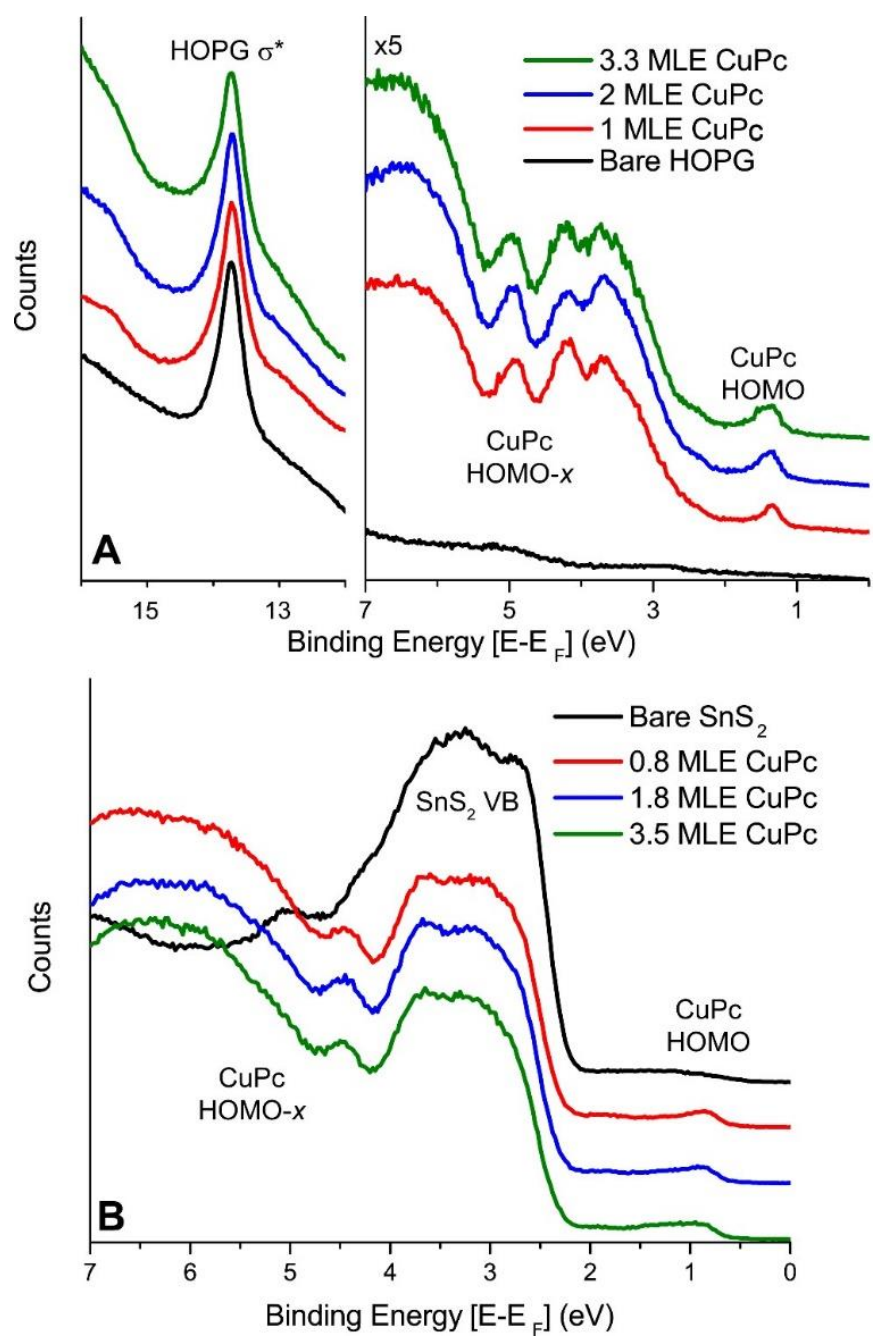

Figure 1: Evolution of the UP spectra with CuPc coverage for (A) HOPG and (B) SnS 2 . Spectra are offset for clarity.

Next, we consider the evolution of the workfunction, which we use as a first indicator for interfacial interactions. As can be seen in Figure 2A, the workfunction of CuPc/HOPG changes minimally with CuPc coverage, in agreement with previous studies $[11,12,39]$. The interface dipole developed at this interface is thus negligible. This is expected from the absence of a molecular dipole moment, the low DOS near the Fermi energy for HOPG, and the weak interfacial interactions on this surface. In contrast, for $\mathrm{CuPc} / \mathrm{SnS}_{2}$ the workfunction changes considerably with CuPc coverage, decreasing by 0.23(3) eV at only 0.5 MLE CUPc. This workfunction decrease, saturating near $2 \mathrm{MLE}$, is clearly seen in the shift of the secondary electron cutoff, as shown in Figure 2B. Based on a previous report for the 
related system of $\mathrm{VONc} / \mathrm{SnS}_{2}$ [34], this workfunction change is composed of a combination of a developing interface dipole (ID) and substrate band bending (BB). While we address each of these aspects below in detail, we may already conclude that there are significant electronic interactions at the $\mathrm{CuPc} / \mathrm{SnS}_{2}$ interface, contrary to expectations that might result from a naïve inspection of the $\mathrm{SnS}_{2}$ band structure and its low DOS near the Fermi energy.
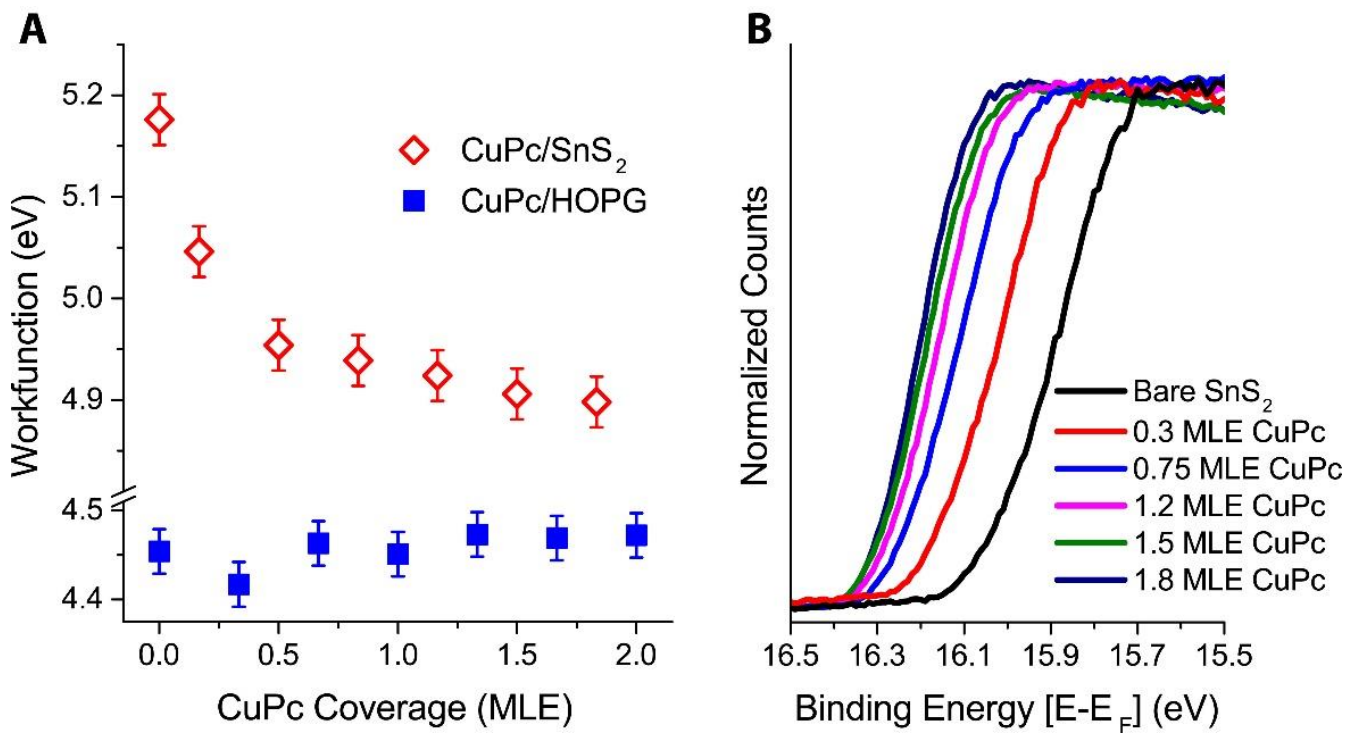

Figure 2: (A) Workfunction evolution as a function of CuPc coverage on both $\mathrm{SnS}_{2}$ and HOPG. (B) Spectral evolution of the secondary electron cutoff region for $\mathrm{CuPc} / \mathrm{SnS}_{2}$.

We next consider how the different substrates influence the electronic properties of the CuPc molecules. As shown in Figure 3A, the evolution of the binding energy of the HOMO of CuPc, determined here as the peak centroid for the sake of simplicity, exhibits substrate-dependent behavior that is indicative of differential interfacial interactions. On HOPG, CuPc is known to transition from wetting layer growth to growth of bilayer islands before completion of the first monolayer [39]. This change in growth mode is accompanied by a shift of the HOMO binding energy, observed here at approximately 0.6 MLE (Figure 3B). Since HOPG interacts weakly with CuPc [8,46-48], this relatively abrupt change for CuPc/HOPG, as compared to $\mathrm{CuPc}_{\mathrm{SnS}}$, reflects differential screening of the photohole and modification of the substrate image potential $[25,49]$. This is in agreement with the 
growth mode of CuPc on HOPG, where the tendency of CuPc to aggregate above 0.5 MLE indicates that there is significant intermolecular interaction and weak molecule/substrate coupling [50-52]. No further changes are observed in the molecular electronic structure on HOPG once bilayer and eventually islandtype growth set in.
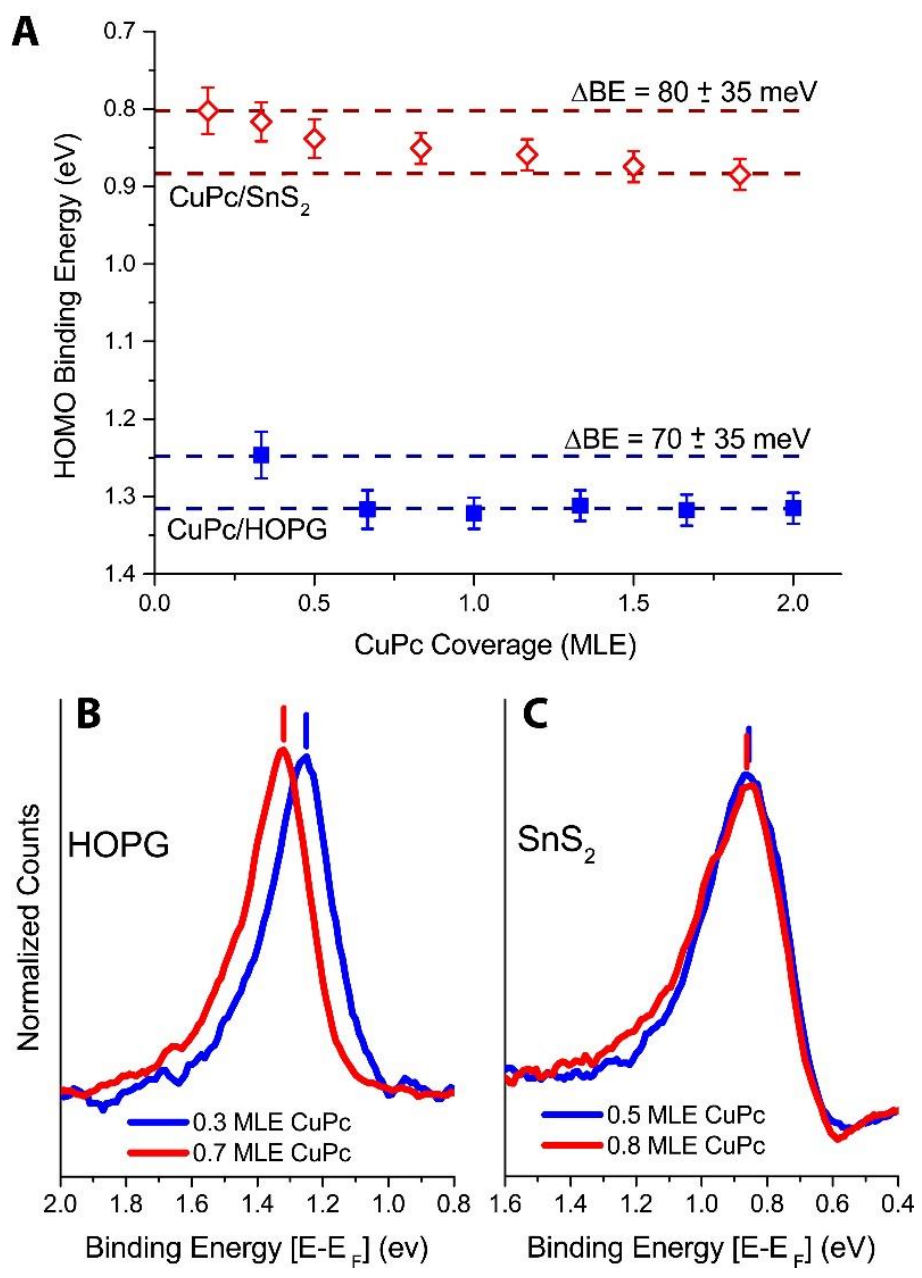

Figure 3: (A) Plot of highest occupied molecular orbital binding energy as a function of CuPc coverage for both HOPG and $\mathrm{SnS}_{2}$. (B) Background-subtracted UP spectra of the CuPc HOMO before and after the transition from monolayer to bilayer island growth on HOPG. (C) Background-subtracted UP spectra of the CuPc HOMO on $\mathrm{SnS}_{2}$.

No such binding energy switch is observed for CuPc on $\mathrm{SnS}_{2}$ (Figure 3C). Instead, the HOMO shifts continuously to higher binding energies with coverage (Figure $3 \mathrm{~A}$ ), and a discrete transition in growth mode cannot be detected spectroscopically. While there are at present no comprehensive 
growth studies of Pcs on $\mathrm{SnS}_{2}$, this observation suggests that polarization energy effects for the $\mathrm{SnS}_{2}$ surface and CuPc films are quite comparable, given the likely island-type growth mode on this surface. Additionally, weak screening in the $\mathrm{SnS}_{2}$ substrate also reduces coverage-dependent image potential effects [49]. Since the CuPc/SnS 2 interface does not display strong changes in polarization energy or image potential with coverage, the observed binding energy shift of the CuPc HOMO must be induced by band bending in the $\mathrm{SnS}_{2}$ substrate. As the coverage of CuPc on $\mathrm{SnS}_{2}$ increases, the chemical potential is raised towards the conduction band minimum, giving rise to a slow decrease in the binding energy of all observed features. In contrast, band bending cannot occur for CuPc/HOPG, and consequently the binding energy of the CuPc HOMO does not change significantly for coverages greater than 0.5 MLE.

We use the spectral evolution of the $\mathrm{SnS}_{2}$ valence band (VB) features above $2 \mathrm{eV}$ to evaluate quantitatively the band bending contribution at the $\mathrm{CuPc}_{\mathrm{SnS}}$ interface. The VB is dominated by the $\mathrm{S}$ $3 p$ band, which can be identified clearly in UPS. Figure 4A shows that the $\mathrm{SnS}_{2}$ VB features shift indeed with CuPc coverage, a hallmark of band bending. We note also that the CuPc HOMO tracks the $\mathrm{SnS}_{2}$ VB features exactly over the complete coverage range (Figure 4B), indicating that band bending occurs exclusively within the $\mathrm{SnS}_{2}$ phase. This is expected given the low coverage regime investigated here, which does not support a sufficient density of defect states in the organic film. Significant band bending within the organic phase can be observed in organic thin films in general $[42,53]$ and in CuPc films in particular [54-56]; such effects are however only supported at coverages in excess of 10 MLE. Furthermore, previous work on the related system of $\mathrm{VONc} / \mathrm{SnS}_{2}$ also revealed that significant band bending was confined to $\mathrm{SnS}_{2}$ for the first few monolayers [34]. Finally, molecular adsorption has been demonstrated to dope $\mathrm{WS}_{2}$ [57], a closely related TMD, consequently leading to band bending. Importantly, we note that the binding energy shift of the CuPc HOMO reported in Figure $3 \mathrm{~A}$ appears to be induced entirely and purely by substrate band bending. Therefore, band bending in $\mathrm{SnS}_{2}$ constitutes the main driving force for the evolution of the molecular electronic structure. Since the CuPc 
$\mathrm{HOMO}$ is quite narrow and at an energy quite different from either valence or conduction band of $\mathrm{SnS}_{2}$, band bending in $\mathrm{SnS}_{2}$ must involve states located inside the band gap [34], reinforcing the importance of substrate DOGS for energy level alignment.
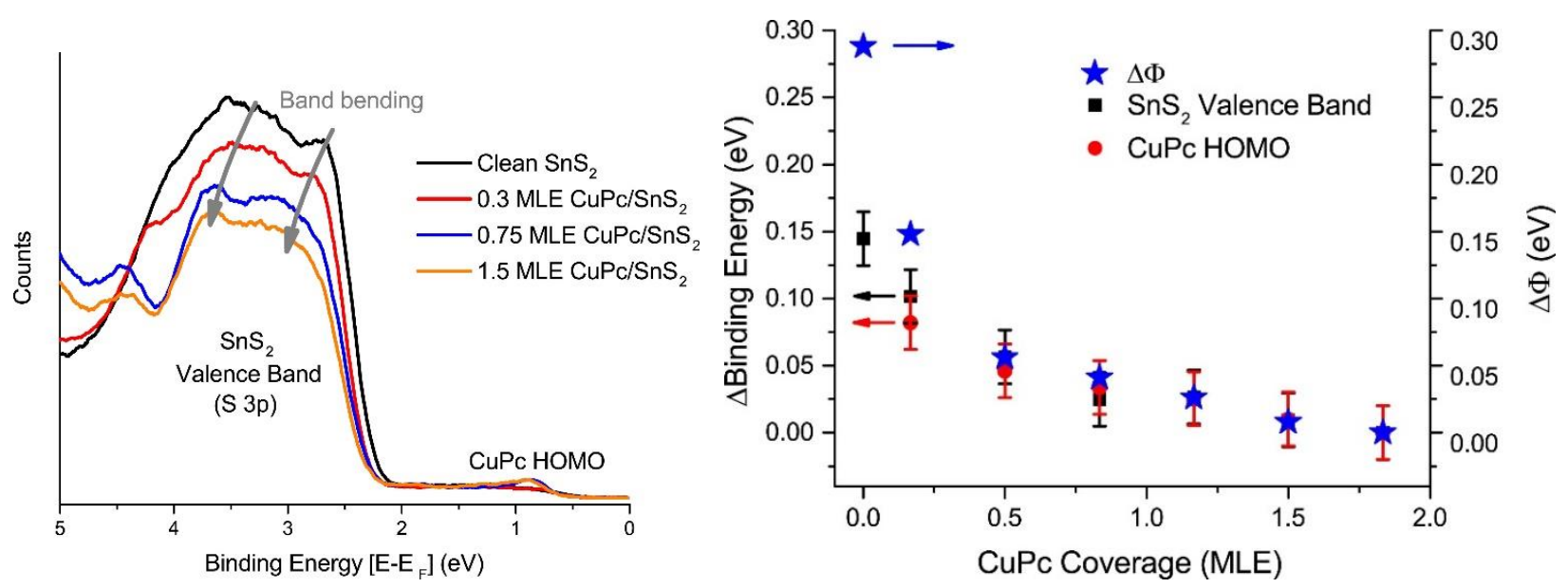

Figure 4: (A) UP spectra of the $\mathrm{SnS}_{2}$ valence band, indicating the downward band bending induced in the substrate upon adsorption of CuPc. (B) Change in $\mathrm{SnS}_{2}$ workfunction $(\Delta \Phi)$ and binding energy of the highest lying $\mathrm{SnS}_{2} \mathrm{~S} 3 p$ VB feature and CuPc HOMO, as a function of CuPc coverage. For coverages in excess of $0.5 \mathrm{MLE}$, all three observables change by the same amount within experimental accuracy. For coverages less than $0.5 \mathrm{MLE}$, a significant interface dipole is present as well.

We emphasize that the interfacial energy level alignment on both substrates is predominantly driven by electronic interactions rather than differences in film structure: Molecular growth modes on HOPG and TMDs have been demonstrated to be similar, as expected for van der Waals-type surfaces [41-44]. Furthermore, this is in agreement with the fact that our experimental observations cannot be explained satisfactorily by differences in film growth alone. The significant workfunction drop induced by CuPc adsorption on $\mathrm{SnS}_{2}$ reveals a strong interface dipole in excess of $200 \mathrm{meV}$, not expected for a nonpolar molecule in the absence of interfacial electronic coupling or a large density of surface electrons. The latter is found commonly e.g. on certain surfaces of coinage metals and gives rise to exchange correlation effects also referred to as Pauli pushback [58], but is missing for both $\mathrm{SnS}_{2}$ and HOPG. The suggested similar growth mode on both surfaces is also supported by closely matching peak shape and widths of the CuPc HOMO (Figure 3B), aside from a very small multilayer peak for $\mathrm{CuPc}_{\mathrm{SnS}}$ 
that appears at higher binding energy, around $1.2 \mathrm{eV}$. In summary, the evolution of the CuPc HOMO binding energy in particular and of the interfacial electronic structure on both substrates is primarily driven by electronic interactions. On $\mathrm{SnS}_{2}$, the dominant factor is substrate band bending, while on HOPG it is polarization energy and image potential effects.

Further insight can be gained by examining the very lowest coverages of CuPc on $\mathrm{SnS}_{2}$. At this interface, an interface dipole is developed that dominates the large drop in workfunction, much larger than the effects of substrate band bending. The interface dipole is already established for the very thinnest CuPc films and saturates at 0.5 MLE coverage. We attribute this observation to two separate phenomena during thin film growth: At the lowest coverages, CuPc molecules are known to nucleate primarily at step edges and defect sites on metal dichalcogenides $[17,52,59]$, such that they likely dominate at these binding sites and at low molecular coverages. Such undercoordinated sites induce strong surface/molecule electronic interactions, inducing hybrid gap states that are likely primarily responsible for the interface dipole at low coverages. At higher coverages, in the event of StranskiKrastanov- or other island-type growth, the workfunction exhibits a weaker dependence on CuPc coverage, since the CuPc islands grow largely vertically.

In order to describe this interface dipole in more detail, we extract the CuPc ionization energy as a function of coverage (Figure 5). For HOPG, since the workfunction changes minimally with the adsorption of CuPc, the shift to higher CuPc ionization energy reflects the monolayer to bilayer island transition and the associated change in polarization energies. In contrast, the $\mathrm{CuPc} / \mathrm{SnS}_{2}$ sample shows the opposite behavior, i.e. the ionization energy decreases as the coverage of $\mathrm{CuPc}$ is increased. Thus, the CuPc wetting layer is energetically stabilized on $\mathrm{SnS}_{2}$, revealing significant electronic coupling across the hybrid interface. The origin of this coupling will be described in more detail in the Discussion. For coverages greater than $0.6 \mathrm{MLE}$, the CuPc ionization energy is however independent of the substrate within experimental resolution. This close agreement in the molecular electronic structure for thicker 
CuPc films on both HOPG and $\mathrm{SnS}_{2}$ indicates that the electronic interactions for the $\mathrm{CuPc} / \mathrm{SnS}_{2}$ interface are confined to very low coverages. After growth of an incomplete wetting layer, the CuPc electronic structure is not perturbed further by the $\mathrm{SnS}_{2}$ surface.

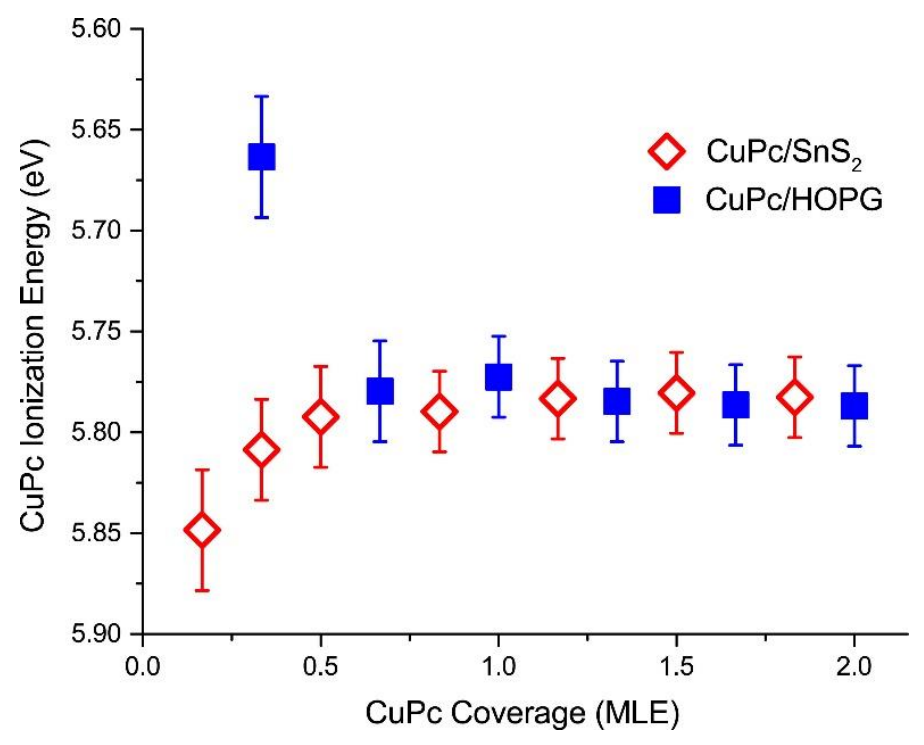

Figure 5: Plot of ionization energy of CuPc as a function of coverage for both HOPG and $\mathrm{SnS}_{2}$. Low coverages of CuPc exhibit significant differences in ionization energy, indicating different interactions across the hybrid interface, while the ionization energy for thicker films is independent of substrate identity, as expected for inert substrate surfaces.

\section{$\underline{\text { Discussion }}$}

Our results suggest that the observed differences in the evolution of the interfacial electronic structure cannot be explained by differences in the CuPc film structure alone. The combination of band bending, interface dipole, and a significantly perturbed CuPc ionization energy indicate instead that significant electronic interactions are occurring at the hybrid semiconductor interface. The relative energies of the $\mathrm{SnS}_{2}$ bands in relation to the CuPc HOMO clearly point to states within the $\mathrm{SnS}_{2}$ band gap as the origin of these interactions. We will now discuss the importance of substrate gap states and their effect on the interface in more detail. 
Previous work has established that the Schottky model of vacuum level alignment is sufficient to understand cases where the workfunction of an inert substrate is significantly larger than the ionization energy of the molecular adsorbate $[14,23]$. In that case, vacuum level of substrate and organic thin film align, leading to a minimal interface dipole. Although this captures the situation for CuPc/HOPG, the interface of CuPc with $\mathrm{SnS}_{2}$ violates this trend. The measured CuPc ionization energy of 5.78(2) eV, in agreement with established values $[8,11]$, is substantially larger than the $\mathrm{SnS}_{2}$ workfunction of 5.20(3) eV. Nevertheless, the CuPc/SnS 2 interface develops a significant dipole upon adsorption of CuPc, revealed as a vacuum level offset already observed at rather low coverages. Clearly, additional considerations beyond those of the Schottky model must be included in order to understand the observed interfacial energy level alignment, suggesting that hybrid organic/inorganic semiconductor interfaces differ from organic/metal interfaces in significant ways.

In order to explore this conclusion further, it is important to consider the possibility of extensive HOMO band tailing towards the Fermi energy. Charge-transfer during contact formation from the substrate to the tail density of states above $\underline{E}_{E}$ has been suggested to be a principal cause for Fermi level pinning $[3,13,60]$. Merely comparing peak-widths and -energies from UPS may be insufficient to assess the extent to which such tail states exist, as it is by now well established that the effective width of the molecular HOMO DOS can be substantially larger than that measured by UPS $[5,6,14,23,32]$. Since a significant interface dipole can be established with only a fraction of one electronic charge per molecule transferred across the interface, the substrate Fermi level may pin the upper edge of the tailing HOMO DOS. This DOS is small and thus often hard to detect in a typical photoemission experiment [33]. In our case however, we can rule out CuPc band tailing above $\underline{E}_{\underline{F}}$ as the source for the observed energy level alignment by comparing $\mathrm{CuPc} / \mathrm{SnS}_{2}$ with the related system of $\mathrm{VONc} / \mathrm{SnS}_{2}$. Note that naphthalocyanine films are structurally closely related to phthalocyanine films, making a direct comparison of the two systems possible $[43,61]$. As seen in Figure 6 , the HOMO of VONc lies much closer to the Fermi edge on 
$\mathrm{SnS}_{2}$, with a peak onset at approximately $0.16(3) \mathrm{eV}$. In contrast, the onset of the CuPc HOMO is at $0.65(3) \mathrm{eV}$, substantially deeper inside the bandgap of $\mathrm{SnS}_{2}$. This difference corresponds almost exactly to the difference in ionization energy between CuPc and VONc ( 5.3 eV for a 0.5 MLE VONc film on HOPG [62]). Neither VONc nor CuPc are thus strongly pinned to the $\mathrm{SnS}_{2}$ substrate Fermi level, and tail states in the CuPc film only contribute to a minor degree to the energy level alignment with $\mathrm{SnS}_{2}$. Rather, the interfacial electronic structure for these systems must be driven by interactions with $\mathrm{SnS}_{2}$ gap states [34]. Conventional models used to understand organic semiconductor interfaces are thus insufficient for CuPc/SnS 2 in particular and likely hybrid interfaces in general $[63,64]$.

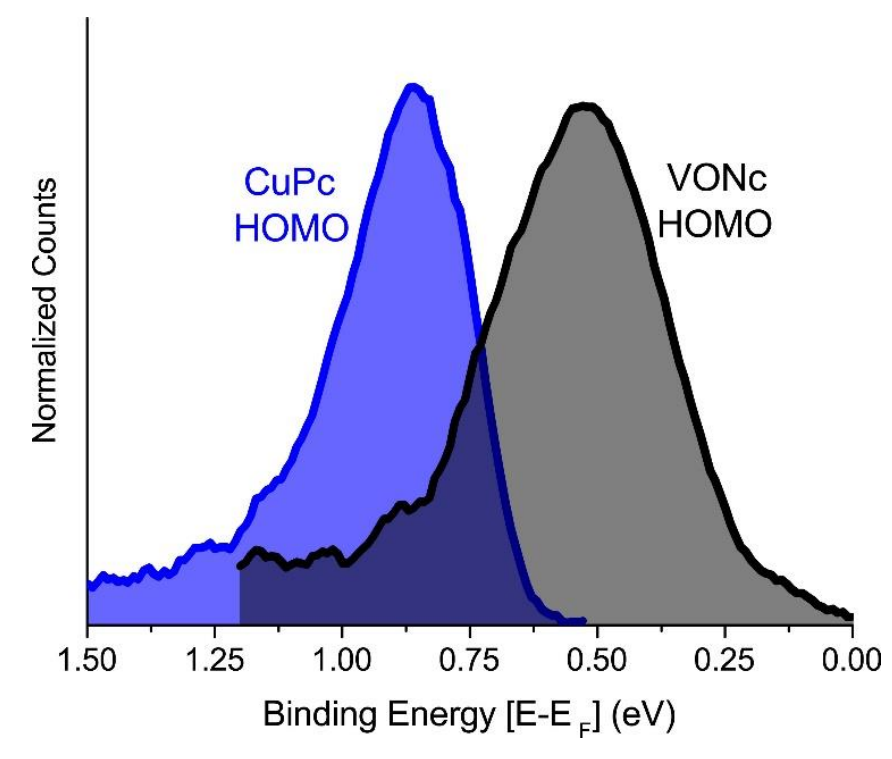

Figure 6: UP spectra of the HOMO for $1 \mathrm{MLE}$ VONc or CuPc deposited on $\mathrm{SnS}_{2}$, demonstrating the lack of Fermi level pinning in the case of $\mathrm{CuPc} / \mathrm{SnS}_{2}$. Contributions from the Hel( $\left.\beta\right)$ satellite have been subtracted.

Based on these considerations and the fact that the CuPc HOMO is located well within the $\mathrm{SnS}_{2}$ bandgap, we conclude that the interfacial interactions must result from electronic coupling between the CuPc HOMO and states within the $\mathrm{SnS}_{2}$ band gap. This is supported by the observation that the CuPc ionization energy differs most strongly between the two interfaces for the lowest coverages (Figure 5), i.e. in the coverage regime where the molecules interact most directly with the surface and hence with 
reactive sites in the substrate. The $\mathrm{SnS}_{2}$ surface hosts thus a small but important DOGS in the nearsurface region that alters the interfacial energy level alignment. Both island growth and the finite density of the substrate gap states result in the convergence of the ionization energies on both substrates, i.e. following "saturation" of the reactive DOGS, the CuPc ionization energy is determined primarily by the structure of the molecular film. Our experimental evidence points therefore to electronic coupling at the $\mathrm{CuPc} / \mathrm{SnS}_{2}$ interface, not film structure, as the dominant source of the observed energy level alignment.

An "empty band gap" model of the $\mathrm{SnS}_{2}$ semiconductor is evidently insufficient for understanding the interfacial electronic structure at this hybrid interface. The question remains however as to the identity of this DOGS, and more specifically, if the electronic interactions at this interface are merely a result of the extrinsic $\mathrm{n}$-doping of $\mathrm{SnS}_{2}$. In fact, dopant levels from $\mathrm{Cl}^{-}$doping of $\mathrm{SnS}_{2}$ are present in insufficient densities to support the observed electronic structure evolution. This can be estimated from the observed degree of band bending: Adsorption of 1 MLE of CUPc is accompanied by downward band bending of $120 \mathrm{meV}$ (Figure 4B), induced by a surface density of charges $\rho_{S S}$ that is estimated crudely as follows [65]:

$$
\Delta \varphi_{B B}=\frac{e\left(\rho_{S S}\right)^{2}}{2 \varepsilon_{r} \varepsilon_{0} N_{D}}
$$

where $\Delta \varphi_{B B}$ is the band bending magnitude, $e$ is the electron charge, $\varepsilon_{r}$ is the relative permittivity of $\mathrm{SnS}_{2}, \varepsilon_{0}$ is the permittivity of vacuum, and $\underline{\mathrm{N}_{\mathrm{D}}}$ is the dopant density of $\mathrm{SnS}_{2}$. This results in a charged surface state density $\left(\rho_{S S}\right)$ for $\mathrm{CuPc} / \mathrm{SnS}_{2}$ of $4.3 \times 10^{11} \mathrm{~cm}^{-2}$. Based on the known $\mathrm{Cl}^{-}$dopant concentration of $2.3 \times 10^{17} \mathrm{~cm}^{-3}$ [45], and an $\mathrm{SnS}_{2}$ layer thickness of $7 \AA$, we estimate a $\mathrm{Cl}^{-}$density in the surface layer of $\mathrm{SnS}_{2}$ of $2 \times 10^{10} \mathrm{~cm}^{-2}$. The weak interlayer coupling of $\mathrm{SnS}_{2}$ prevents the interaction of deeper-lying $\mathrm{Cl}^{-}$ dopants with the molecules $[66,67]$. The $\mathrm{Cl}^{-}$concentration in the near-surface region is thus more than one order of magnitude smaller than the value necessary for the observed extent of band bending. $\mathrm{Cl}$ dopants are therefore not sufficient to establish the interfacial energy level alignment of $\mathrm{CuPc} / \mathrm{SnS}_{2}$. 
Alternatively, $\mathrm{S}$ vacancies $\left(\mathrm{S}_{\mathrm{V}}\right)$ should be considered. Previous reports have implicated such point defects as a critical component for the catalytic activity of metal dichalcogenides $[68,69]$, demonstrating clearly that they are electronically active. $\mathrm{S}_{v}$ have been directly observed in $\mathrm{SnS}_{2}$ and other TMDs with both high-resolution transmission electron microscopy and scanning tunneling microscopy [52,70-73]. Other defects, such as sulfur divacancies and tin vacancies, are also present, but are comparatively scarce and energetically unstable in comparison to $S_{V}[72]$, resulting likely in minimal impact on the interfacial energy level alignment. In contrast, $\mathrm{S}_{V}$ play a significant role in the photoinjection yields observed for molecular dyes on $\mathrm{SnS}_{2}$ [52], and in determining charge transport properties of TMDs $[71,74]$. Both computational and experimental work has shown that $S_{v}$ create localized electronic states within the bandgap of TMDs $[71,73,75]$. In the case of $\mathrm{MoS}_{2}, \mathrm{~S}_{\mathrm{V}}$ induce both occupied and unoccupied gap states [73]. We conclude thus that $S_{V}$ enable complex interfacial electronic interactions as observed in this paper and elsewhere [34]. This conclusion is also in agreement with a previous report showing that the energy levels of atomic adsorbates are pinned within the bandgap of GaAs by vacancy defects [76]. Perhaps most strikingly and of importance for rational design of efficient hybrid organic/inorganic optoelectronic devices, it is important to realize that the estimated surface density of CuPc molecules is approximately $5 \times 10^{13} \mathrm{~cm}^{-2}$ [9], well over an order of magnitude larger than the surface concentration of the charged substrate gap states. Therefore, even though the DOGS significantly influences the interfacial energy level alignment, it is rather small and likely difficult to observed directly with standard spectroscopic approaches.

\section{Conclusion}

Overall, it is clear that substrate gap states play a significant role in influencing the properties of semiconductor interfaces. Our work expands on the previously established importance of gap states in molecular semiconductor films by highlighting the importance of gap states in the inorganic substrate as 
well. Indeed, gap states in metal dichalcogenides have been shown to significantly affect carrier mobility $[36,37,77]$, dynamic shifts in energy level alignment [34], and electronic interactions between stacked van der Waals materials [38]. The high degree of control in inorganic semiconductor growth, the structural similarity of layered van der Waals materials, and the considerable understanding developed for prototypical organic semiconductor interfaces with such materials opens an avenue for controlled exploration of the role of gap states for organic interfaces. These effects must be understood at a fundamental level in order to enable next-generation technologies that incorporate hybrid organic/inorganic semiconductor interfaces in general, or metal dichalcogenides surfaces in particular.

\section{Acknowledgment}

Support from the National Science Foundation under grant number CHE-1213243 is gratefully acknowledged. We would also like to thank Prof. Bruce Parkinson (Wyoming) for providing the $\mathrm{SnS}_{2}$ crystals. 


\section{References}

[1] S. Braun, W.R. Salaneck, M. Fahlman, Adv. Mater. 21 (2009) 1450.

[2] O.L.A. Monti, J. Phys. Chem. Lett. 3 (2012) 2342.

[3] M. Oehzelt, N. Koch, G. Heimel, Nat. Commun. 5 (2014) 4174.

[4] J. Hwang, A. Wan, A. Kahn, Mater. Sci. Eng. R Rep. 64 (2009) 1.

[5] T. Hosokai, H. Machida, A. Gerlach, S. Kera, F. Schreiber, N. Ueno, Phys. Rev. B 83 (2011) 195310.

[6] F. Bussolotti, J. Yang, A. Hinderhofer, Y. Huang, W. Chen, S. Kera, A.T.S. Wee, N. Ueno, Phys. Rev. B 89 (2014) 115319.

[7] K. Kamiya, M. Momose, A. Kitamura, Y. Harada, N. Ueno, S. Hasegawa, T. Miyazaki, H. Inokuchi, S. Narioka, H. Ishii, K. Seki, J. Electron Spectrosc. Relat. Phenom. 76 (1995) 213.

[8] S. Kera, H. Yamane, I. Sakuragi, K.K. Okudaira, N. Ueno, Chem. Phys. Lett. 364 (2002) 93.

[9] K. Kamiya Okudaira, S. Hasegawa, H. Ishii, K. Seki, Y. Harada, N. Ueno, J. Appl. Phys. 85 (1999) 6453.

[10] N. Ueno, K. Suzuki, M. Momose, M. Kushida, K. Sugita, Jpn. J. Appl. Phys. 33 (1994) 319.

[11] S. Kera, Y. Yabuuchi, H. Yamane, H. Setoyama, K. Okudaira, A. Kahn, N. Ueno, Phys. Rev. B 70 (2004) 085304.

[12] T. Sugiyama, T. Sasaki, S. Kera, N. Ueno, T. Munakata, Appl. Phys. Lett. 89 (2006) 202116.

[13] A. Kahn, N. Koch, W. Gao, J. Polym. Sci. Part B Polym. Phys. 41 (2003) 2529.

[14] N. Koch, N. Ueno, A.T.S. Wee, eds., The Molecule-Metal Interface, Wiley-VCH Verlag GmbH \& Co. KGaA, Weinheim, Germany, 2013.

[15] A. Terentjevs, M.P. Steele, M.L. Blumenfeld, N. Ilyas, L.L. Kelly, E. Fabiano, O.L.A. Monti, F. Della Sala, J. Phys. Chem. C 115 (2011) 21128.

[16] W. Liu, A. Tkatchenko, M. Scheffler, Acc. Chem. Res. 47 (2014) 3369.

[17] L.K. Chau, C. Arbour, G.E. Collins, K.W. Nebesny, P.A. Lee, C.D. England, N.R. Armstrong, B.A. Parkinson, J. Phys. Chem. 97 (1993) 2690.

[18] K. Kamiya, M. Momose, Y. Harada, N. Ueno, T. Miyazaki, S. Hasegawa, H. Inokuchi, S. Narioka, H. Ishii, K. Seki, Mol. Cryst. Liq. Cryst. Sci. Technol. Sect. Mol. Cryst. Liq. Cryst. 267 (1995) 211.

[19] A. Schmidt, R. Schlaf, D. Louder, L.-K. Chau, S.-Y. Chen, T. Fritz, M.F. Lawrence, B.A. Parkinson, N.R. Armstrong, Chem. Mater. 7 (1995) 2127.

[20] S. Duhm, G. Heimel, I. Salzmann, H. Glowatzki, R.L. Johnson, A. Vollmer, J.P. Rabe, N. Koch, Nat. Mater. 7 (2008) 326.

[21] N. Ilyas, O.L.A. Monti, Phys. Rev. B 90 (2014) 125435.

[22] M. Momose, K. Kamiya, K. Sugita, N. Ueno, Jpn. J. Appl. Phys. 33 (1994) 4754.

[23] H. Fukagawa, S. Kera, T. Kataoka, S. Hosoumi, Y. Watanabe, K. Kudo, N. Ueno, Adv. Mater. 19 (2007) 665.

[24] M.P. Steele, M.L. Blumenfeld, O.L.A. Monti, J. Chem. Phys. 133 (2010) 124701.

[25] M.P. Steele, L.L. Kelly, N. Ilyas, O.L.A. Monti, J. Chem. Phys. 135 (2011) 124702.

[26] O.T. Hofmann, D.A. Egger, E. Zojer, Nano Lett. 10 (2010) 4369.

[27] A. Natan, L. Kronik, H. Haick, R.T. Tung, Adv. Mater. 19 (2007) 4103.

[28] T. Kataoka, H. Fukagawa, S. Hosoumi, K. Nebashi, K. Sakamoto, N. Ueno, Chem. Phys. Lett. 451 (2008) 43.

[29] S. Kera, H. Setoyama, M. Onoue, K. Okudaira, Y. Harada, N. Ueno, Phys. Rev. B 63 (2001) 115204.

[30] I.G. Hill, J. Schwartz, A. Kahn, Org. Electron. 1 (2000) 5.

[31] C. Shen, A. Kahn, Org. Electron. 2 (2001) 89.

[32] T. Sueyoshi, H. Fukagawa, M. Ono, S. Kera, N. Ueno, Appl. Phys. Lett. 95 (2009) 183303.

[33] T. Sueyoshi, H. Kakuta, M. Ono, K. Sakamoto, S. Kera, N. Ueno, Appl. Phys. Lett. 96 (2010) 093303.

[34] D.A. Racke, O.L.A. Monti, Surf. Sci. 630 (2014) 136.

[35] T. Chassé, C.-I. Wu, I.G. Hill, A. Kahn, J. Appl. Phys. 85 (1999) 6589. 
[36] W. Zhu, T. Low, Y.-H. Lee, H. Wang, D.B. Farmer, J. Kong, F. Xia, P. Avouris, Nat. Commun. 5 (2014) 3087.

[37] C.J. Docherty, P. Parkinson, H.J. Joyce, M.-H. Chiu, C.-H. Chen, M.-Y. Lee, L.-J. Li, L.M. Herz, M.B. Johnston, ACS Nano 8 (2014) 11147.

[38] M.-H. Chiu, M.-Y. Li, W. Zhang, W.-T. Hsu, W.-H. Chang, M. Terrones, H. Terrones, L.-J. Li, ACS Nano 8 (2014) 9649.

[39] T. Shimada, K. Hamaguchi, A. Koma, F.S. Ohuchi, Appl. Phys. Lett. 72 (1998) 1869.

[40] T. Munakata, T. Sugiyama, T. Masuda, M. Aida, N. Ueno, Appl. Phys. Lett. 85 (2004) 3584.

[41] P.G. Schroeder, M.W. Nelson, B.A. Parkinson, R. Schlaf, Surf. Sci. 459 (2000) 349.

[42] P.G. Schroeder, C.B. France, B.A. Parkinson, R. Schlaf, J. Appl. Phys. 91 (2002) 9095.

[43] C. Ludwig, R. Strohmaier, J. Petersen, B. Gompf, W. Eisenmenger, J. Vac. Sci. Technol. B Microelectron. Nanometer Struct. 12 (1994) 1963.

[44] Y.L. Huang, H. Li, J. Ma, H. Huang, W. Chen, A.T.S. Wee, Langmuir 26 (2010) 3329.

[45] L. Sharp, D. Soltz, B.A. Parkinson, Cryst. Growth Des. 6 (2006) 1523.

[46] H. Fukagawa, S. Hosoumi, H. Yamane, S. Kera, N. Ueno, Phys. Rev. B 83 (2011) 085304.

[47] J. Ren, S. Meng, Y.-L. Wang, X.-C. Ma, Q.-K. Xue, E. Kaxiras, J. Chem. Phys. 134 (2011) 194706.

[48] J.H. Park, P. Choudhury, A.C. Kummel, J. Phys. Chem. C 118 (2014) 10076.

[49] J. Zhao, M. Feng, D.B. Dougherty, H. Sun, H. Petek, ACS Nano 8 (2014) 10988.

[50] J.R. Fryer, R.B. McKay, R.R. Mather, K.S.W. Sing, J. Chem. Technol. Biotechnol. 31 (1981) 371.

[51] R. Litrán, E. Blanco, M. Ramírez-del-Solar, J. Non-Cryst. Solids 333 (2004) 327.

[52] G.E. Collins, J. Vac. Sci. Technol. Vac. Surf. Films 10 (1992) 2902.

[53] R. Schlaf, P.G. Schroeder, M.W. Nelson, B.A. Parkinson, P.A. Lee, K.W. Nebesny, N.R. Armstrong, J. Appl. Phys. 86 (1999) 1499.

[54] M. Gorgoi, D.R.T. Zahn, Org. Electron. 6 (2005) 168.

[55] K.M. Lau, J.X. Tang, H.Y. Sun, C.S. Lee, S.T. Lee, D. Yan, Appl. Phys. Lett. 88 (2006) 173513.

[56] B. Ben Hamada, A. Souissi, S. Menzli, I. Arbi, A. Akremi, C. Chefi, M. Derivaz, Thin Solid Films 567 (2014) 96.

[57] N. Peimyoo, W. Yang, J. Shang, X. Shen, Y. Wang, T. Yu, ACS Nano 8 (2014) 11320.

[58] H. Ishii, K. Sugiyama, E. Ito, K. Seki, Adv. Mater. 11 (1999) 605.

[59] C.D. England, G.E. Collins, T.J. Schuerlein, N.R. Armstrong, Langmuir 10 (1994) 2748.

[60] H. Vázquez, F. Flores, R. Oszwaldowski, J. Ortega, R. Pérez, A. Kahn, Appl. Surf. Sci. 234 (2004) 107.

[61] M. Lackinger, T. Müller, T.G. Gopakumar, F. Müller, M. Hietschold, G.W. Flynn, J. Phys. Chem. B 108 (2004) 2279.

[62] M.L. Blumenfeld, M.P. Steele, N. Ilyas, O.L.A. Monti, Surf. Sci. 604 (2010) 1649.

[63] P. Winget, L.K. Schirra, D. Cornil, H. Li, V. Coropceanu, P.F. Ndione, A.K. Sigdel, D.S. Ginley, J.J. Berry, J. Shim, H. Kim, B. Kippelen, J.-L. Brédas, O.L.A. Monti, Adv. Mater. 26 (2014) 4711.

[64] P. Schulz, L.L. Kelly, P. Winget, H. Li, H. Kim, P.F. Ndione, A.K. Sigdel, J.J. Berry, S. Graham, J.-L. Brédas, A. Kahn, O.L.A. Monti, Adv. Funct. Mater. 24 (2014) 7381.

[65]Z. Zhang, J.T. Yates, Chem. Rev. 112 (2012) 5520.

[66] U. Berg, T. Chassé, Phys. Status Solidi B 135 (1986) 633.

[67] S. Das, J. Appenzeller, Nano Lett. 13 (2013) 3396.

[68] M. Chhowalla, H.S. Shin, G. Eda, L.-J. Li, K.P. Loh, H. Zhang, Nat. Chem. 5 (2013) 263.

[69] D. Le, T.B. Rawal, T.S. Rahman, J. Phys. Chem. C 118 (2014) 5346.

[70] B. Parkinson, J. Am. Chem. Soc. 112 (1990) 7498.

[71] H. Qiu, T. Xu, Z. Wang, W. Ren, H. Nan, Z. Ni, Q. Chen, S. Yuan, F. Miao, F. Song, G. Long, Y. Shi, L. Sun, J. Wang, X. Wang, Nat. Commun. 4 (2013).

[72] W. Zhou, X. Zou, S. Najmaei, Z. Liu, Y. Shi, J. Kong, J. Lou, P.M. Ajayan, B.I. Yakobson, J.-C. Idrobo, Nano Lett. 13 (2013) 2615. 
[73] H.-P. Komsa, S. Kurasch, O. Lehtinen, U. Kaiser, A.V. Krasheninnikov, Phys. Rev. B 88 (2013) 035301. [74] Z. Yu, Y. Pan, Y. Shen, Z. Wang, Z.-Y. Ong, T. Xu, R. Xin, L. Pan, B. Wang, L. Sun, J. Wang, G. Zhang, Y.W. Zhang, Y. Shi, X. Wang, Nat. Commun. 5 (2014) 5290.

[75] S. Yuan, R. Roldán, M.I. Katsnelson, F. Guinea, Phys. Rev. B 90 (2014) 041402(R).

[76] L. Brillson, Surf. Sci. Rep. 2 (1982) 123.

[77] A.K. Singh, S. Andleeb, J. Singh, H.T. Dung, Y. Seo, J. Eom, Adv. Funct. Mater. (2014) 7125. 